\title{
Immersive VR in Phantom Limb Pain Therapy of Amputee Patients Due to Critical Limb Ischemia
} \author{
Mironiuc ${ }^{4}$ \\ 1 County Clinical Emergency Hospital of Cluj-Napoca, Romania \\ 2 Individual Office of Clinical Psychology and Psychotherapy, Cluj-Napoca, Romania \\ 3 "Niculae Stancioiu" Heart Institute of Cluj-Napoca, Romania \\ 4 University of Medicine and Pharmacie "Iuliu Hatieganu", Cluj-Napoca, Romania
}

Ana-Maria Zanfir ${ }^{*}$, Domnita llea Georgescu², Sergiu Cristian Turturica ${ }^{3}$, Mihaela Eczedi ${ }^{1}$, Aurel

\begin{abstract}
Introduction: Phantom limb pain (PLP) occurs in approximately $75 \%$ of patients who undergo limb amputation. In identifying the etiopathogenic mechanisms, multidisciplinary approaches are increasingly important in explaining the causality based on neurological and psychological factors. PLP has many negative effects on the amputee's physical and mental integrity, which is why a variety of treatments have been conceived, whose effectiveness is rather limited. Objective: The purpose of this study is to evaluate the effectiveness of an immersive virtual reality (IVR) intervention program using the mirror therapy principles in decreasing PLP. Method: Twenty participants suffering from PLP were randomly assigned to one of the two intervention groups: IVR and kinesiotherapy, respectively. Pre- and post-intervention measurements were performed both on pain level and on several psychological variables: depression and anxiety symptoms, pain catastrophizing, quality of life, body representation and coping strategies. Results: Preliminary data show a significant pain relief in patients in the IVR group compared to those in the kinesiotherapy group. Besides, significant improvement was found in the case of the patients in the IVR group, in terms of life quality improvement, reducing irrational pain catastrophizing-related thoughts and positive coping strategies (positive refocusing and reappraisal). There were no differences identified between the two groups in terms of anxiety and depression symptoms. Despite expectations, patients in the IVR group experienced a significant increase in one negative coping strategy: rumination. Conclusions: The results obtained are advocating the use of IVI intervention as a method phantom limb pain alleviation, with positive consequences on patients' life quality.
\end{abstract}

Keywords: immersive virtual reality, mirror box therapy, phantom limb pain, cognitive and emotional coping strategies

Received 23 July 2017 / Accepted 7 September 2017

\section{Introduction}

Limb amputation is a major loss and has a significant negative impact on the patient's well-being: from loss of independence and restriction of daily activities to isolation, mental health impairment and body image disturbances. In addition, the amputee may experience sensations in the severed limb, phantom limb pain and stump pain. In this study, we will deal with the phantom limb pain, defined as painful sensations referred to the absent limb $[1,2]$.

Several studies suggest that significant phantom limb pain is rare $[3,4,5]$. On the other hand, many other studies show that $60-80 \%$ of amputees experience phantom limb pain [6]. This lack of consistency between studies may be explained, on the one hand, by the fact that PLP incidence was assessed by taking into account only the population that has actively sought medical assistance for their phantom limb pain and, on the other hand, because this type of pain is often underreported by patients, who are generally convinced that pain in an amputated limb only exists in their minds. Even two years after limb loss, approximately $75 \%$ of amputees complain about pain in their phantom limb $[7,8]$.

Phantom limb pain is a complex phenomenon, with underlying theories evolving from mono-disciplinary approaches, based exclusively on neurological mechanisms,

* Correspondence to: Ana-Maria Zanfir

E-mail: dr.anamaria.zanfir@gmail.com to complex approaches that include both neurological mechanisms and psychological factors. Therefore, the need for multidisciplinary interventions is becoming increasingly stringent [9].

Specialized literature lists two primary levels at which phantom limb pain relief interventions are performed: pharmacological administration and psychological, behavioural and physical intervention [10]. Pharmacological treatments use analgesics, antidepressants, neuroleptics, anticonvulsants, opioids, muscle relaxants etc. Although pharmacological interventions appear to be numerous, a systematic review of the studies in this field shows that there is limited evidence supporting their effectiveness in PLP management [11]. On the other hand, where the pain in the phantom limb is linked to several pathological processes, including cortical changes, an intervention program that includes physical, behavioural or psychological components may prove more beneficial. Psychological interventions address in particular the emotional factors involved in the occurrence and persistence of the pain in the phantom limb [12]. Cognitive-behavioural therapy and hypnotherapy appear to provide encouraging evidence to this effect [13]; however, in the absence of controlled clinical trials and the fact that most results are based on case studies only, there is no solid evidence supporting their effectiveness. On the other hand, behavioural interventions have been given a particular attention, partly owing to their 
affordability and reduced costs. Kinesiotherapy, a thereapy where patients make muscle toning movements, has proven to have only limited effectiveness, with positive results being rather recorded in patients with prosthetic limbs [10]. Therefore, new behavioural interventions need to be applied to patients who have not yet been prosthetized, but who are experiencing pain in their phantom limb. Mirrorbox therapy is one of the most common interventions. The therapy uses the principle of visual illusion to change amputee's bodily representation and thus alleviate PLP.

The therapy uses a box containing a mirror. The amputee places his or her intact limb into the box, which allows $\mathrm{him} /$ her to view a reflection of his/her anatomical limb in the visual space occupied by his/her phantom limb, while moving the intact limb to release tension in the phantom limb. However, the mirror box presents a number of inherent limitations: the illusion is tentative, relaying on the patient's maintaining attentional focus on the reflected image as opposed to the moving anatomical limb; otherwise, a simple glance of the amputee at the missing limb would compromise the illusion. Besides, the patient is forced to remain in a restricted, fixed position and to move the anatomical limb in a very narrow space [14]. The interventions based on mirror therapy seem promising, although most of its positive results come from case studies or singlesubject experiments $[15,16,17]$.

The conclusion is that the literature highlights the need for both controlled clinical trials and for improvement in behavioural interventions, so as to overcome the limitations presented by the mirror therapy. Therefore, this study uses an immersive virtual reality intervention program (IVR) based on mirror-box visual illusion principles. By using the virtual space, the visual illusion is enhanced, because the number of disruptors is limited and the movement space is much wider [18].

In this context, our research aims at assessing the effectiveness of an IVR-based intervention program in reducing phantom limb pain. To this end, we have compared the effectiveness of this type of intervention with a standard behavioural program based on kinesiotherapy. Studies show the higher effectiveness of the interventional programs based on visual illusion compared to those based on physical exercise, for which reason the study did not include a placebo or a waiting list type of control group.

Moreover, in order to draw a comprehensive picture of the psychological mechanisms underlying the pain in the phantom limb, measurements of psychological variables were included: depression and anxiety symptoms, coping strategies, pain catastrophizing, body image representation. A series of studies show that depression symptomatology is associated with pain intensity [19] and that depression and anxiety symptoms persist several years after amputation [20]. Also, amputees tend to use negative cognitive and emotional coping strategies, which have a negative impact on the presence and intensity of pain [19,21]. Furthermore, a number of studies have drawn attention to the negative effects of visual illusion and virtual reality therapies, seen as triggers of distorted bodily representation, given that the creation of an illusion of the amputated limb might exacerbate depression symptoms in amputees, causing them to experience persistent phantom limb pain [22].

\section{Method \\ Design}

This study is a controlled clinical trial, based on two study groups: an IVR intervention group and a kinesiotherapy intervention group. The twenty participants were randomly assigned to one or the other of the two groups. This study was approved by University of Medicine and Pharmacy "Iuliu Hatieganu " ethics committee.

\section{Participants}

The subjects were recruited from among patients who were admitted to the Surgery Clinic 2 in Cluj-Napoca between 2014 and 2017 and who had undergone lower limb amputation surgery. Twenty people were selected to participate in this clinical trial: 16 males (80\%) and 4 females (20\%). The participants were aged 49-87 years, most of them living in urban areas (85\%), and they were distributed by the two intervention groups: the IVR group $(\mathrm{n}=10)$ and the kinesiotherapy group $(\mathrm{n}=10)$. Inclusion criterion: reported pain in the phantom limb. Exclusion criteria: neurological conditions or cognitive impairment.

\section{Measurements}

Pain. The McGill Pain Questionnaire (MPQ) [23] was used to evaluate pain in the phantom limb. The phantom limb pain subscale from the Trinity Amputation and Prosthesis Experience Scale (Gallagher, Desmond \& MacLachlan, 2000) was also used.

Irrational cognitions. The Pain Catastrophizing Scale (PCS) [24], was used to assess irrational pain-related cognitions.

Body image. Amputatee Body Image Scale (ABIS) [25], was used to assess the relative presence of concern related to amputee's own body image.

Depression and anxiety symptoms. The Hospital Depression and Anxiety Scale (HADS) [26], was used to assess the severity of depression and anxiety symptoms.

Coping strategies. The Cognitive Emotion Regulation Scale (CERQ) [27], evaluated the presence of positive or negative coping strategies. The scale contains nine subscales: self-blame, acceptance, rumination, positive refocusing, focus on planning, positive reappraisal, putting into perspective, catastrophizing, blaming others.

\section{Working procedure}

Study participants were selected from among patients who suffered amputation in the lower limb in the period 20142017. They were contacted by phone and informed about the study. All those who wanted to participate signed an informed consent form. 
In the first stage of the study, all participants filled in the initial assessment scales (pain, irrational cognitions, body image, depression and anxiety symptoms, coping strategies). The second stage started 1-3 days after the filling in of the questionnaires and consisted of three intervention sessions by each participant in the two groups (IVR and kinesiotherapy, respectively). The sessions were scheduled 1-2 days apart. The third stage was performed 1-3 days after the last intervention session and included post-intervention measurements for all participants.

\section{Treatments}

\section{Kinesiotherapy intervention}

The subjects were given three physiotherapy sessions of 30 minutes each, where they engaged in exercises for muscle toning in the lower amputated limb (leg and thigh);

Posture exercises, designed to release contraction and avoid vicious position of the stump, coupled with toning of the healthy lower limb muscles.

\section{IVR intervention}

Patients were given three IVR-based therapy sessions. The IVR software was developed specifically for this study, using the Unity $3 \mathrm{D}$ platform. The device comprised a pair of $3 \mathrm{D}$ virtual reality glasses for smartphones, fitted with adjustable aspherical lenses, a Bluetooth gamepad for IVR and a Lenovo K6 phone. In each session, the participants performed three repetitive tasks: placing the virtual representation of the phantom limb into a coloured, sequentially-illuminated square, hitting a virtual ball and lifting the leg to $45^{\circ}$ to touch a virtual bar (Figure 1, Figure 2, Figure 3). First the patient was familiarized with the equipment. Hitting a virtual ball was the first task: the patient controlled the simultaneous movement of the virtual limb (by using the joystick) and the stump. The same principle was applied for the other 2 tasks. The sessions lasted $30 \mathrm{~min}-$ utes each (Figure 4, Figure 5).

\section{Statistical analysis}

The entire set of collected data was statistically evaluated and the significance of each intervention was calculated. A mix Analysis of variance (mixt ANOVA) was conducted, where the within groups factor was the time (pre- and post-interventions) and the between groups factor, was the condition (IVR group and kinetotherapy group). The main effect (time* group, group, time) analysis were carried out in SPSS Version 24. Also, Pearson's linear correlations were calculated using SPSS Version 24.

\section{Results}

To test the effects of the interventions on the variables, we resorted to a mixed variance analysis, where time (pre- and post-intervention) was the within-subjects factor, and the group (IVR/ kinesiotherapy) was the between-groups factor.

\section{Pain in the phantom limb}

The results show that pain assessed with the MPQ was lower in the IVR group at post-assessment compared to the kinesiotherapy group. Statistical analysis has revealed a significant time $e^{*}$ group interaction, $\mathrm{F}(1,10)=23.965$, $\mathrm{p}$ $<0.001, \eta^{2}=0.571$. Also, a time effect $\mathrm{F}(1,20)=26.809$, $\mathrm{p}<0.001, \eta^{2}=0.598$ was recorded. There was no group effect $(\mathrm{p}=0.464)$.

\section{Quality of life}

The quality of life measured by TAPES has shown significant post-intervention increase in patients in the IVR group compared to those in the kinesiotherapy group. A significant time* group interaction $\mathrm{F}(1,10)=15.158, \mathrm{p}=$ $0.001, \eta^{2}=0.457$ was recorded, coupled with a time effect $\mathrm{F}(1,20)=23.684, \mathrm{p}<0.001, \eta^{2}=0.586$. The group effect was absent $(\mathrm{p}=0.707)$.

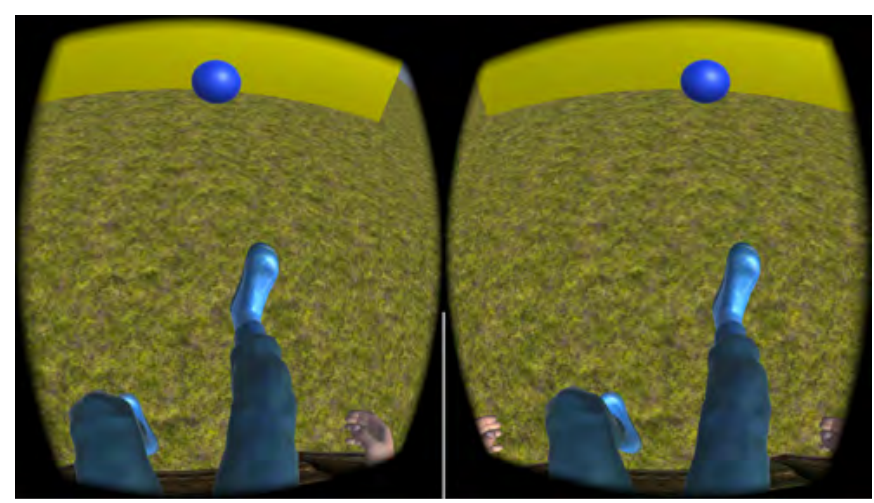

Fig. 1. First exercise - Hitting a virtual ball

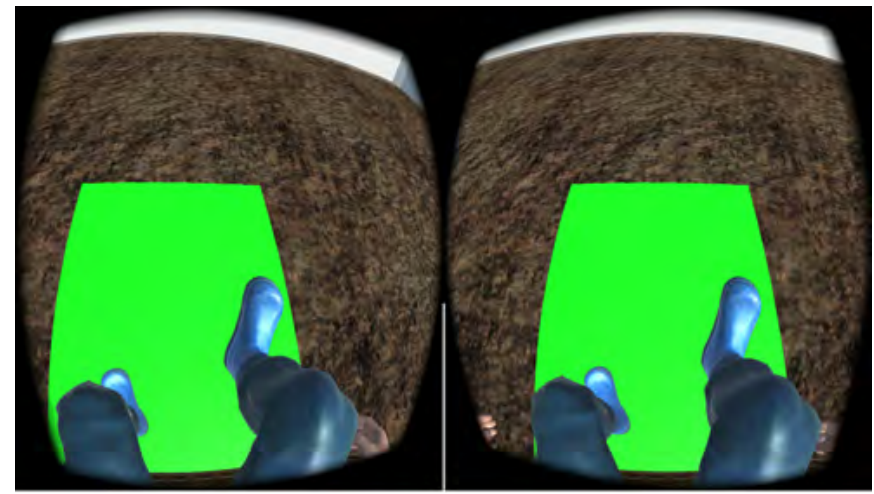

Fig. 2. Second exercise - Placing the virtual representation of the phantom limb into a colored, sequentially-illuminated square

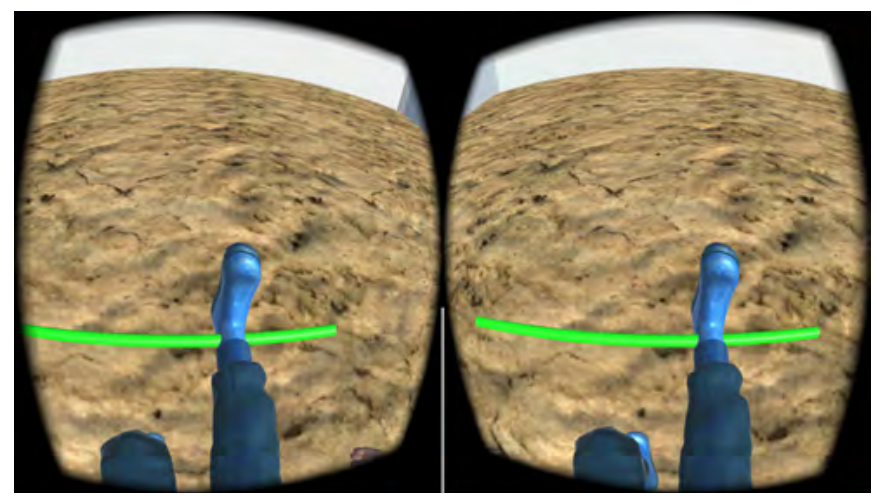

Fig. 3. Third exercise - Lifting the leg to $45^{\circ}$ to touch a virtual bar 


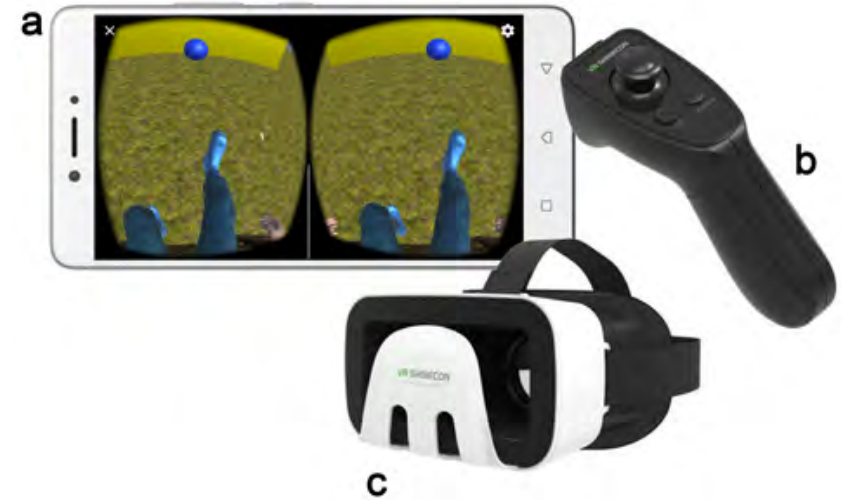

Fig. 4. IVR equipment: a. Lenovo Vibe K6; b. VR Shinecon bluetooth gamepad; c. 3D VR Shinecon glasses for mobile phone

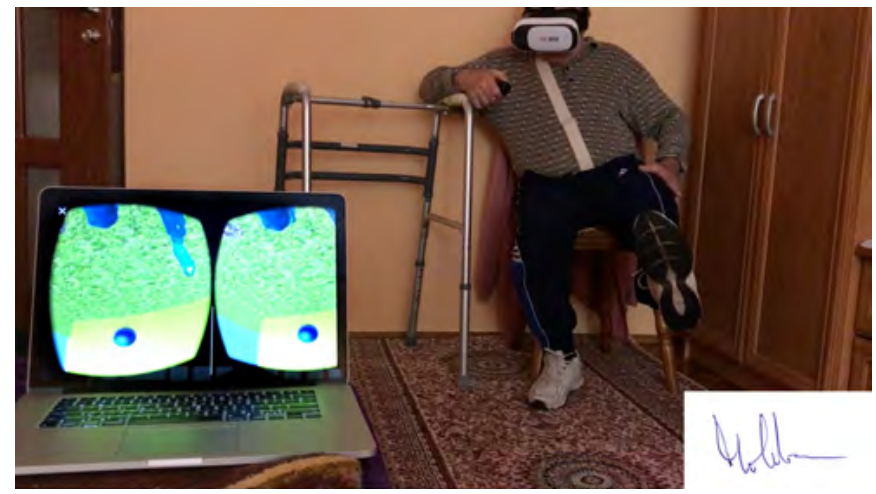

Fig. 5. Patient during IVR session

\section{Depression and anxiety symptoms}

Regarding depression symptoms, a time effect $\mathrm{F}(1,20)=$ $6.560, p=0.02, \eta^{2}=0.267$ was recorded. There was no significant time* group interaction $(\mathrm{p}=0.404)$ and no group effect $(\mathrm{p}=0.605)$.

As far as the anxiety symptoms are concerned, there was a time effect $F(1,20)=9.511, p=0.006, \eta^{2}=0.346$ recorded. No significant time*group interaction $(\mathrm{p}=1)$ and no group effect $(\mathrm{p}=0.941)$ were recorded.

\section{Pain catastrophizing}

The irrational cognitions related to pain catastrophizing were lower in subjects in the IVR group at post-assessment compared to those in the kinesiotherapy group. A significant time* group interaction, $\mathrm{F}(1,10)=12.975, \mathrm{p}=$ $0.002, \eta^{2}=0.419$, was recorded. Also, a significant time effect, $\mathrm{F}(1,20)=18.845, \mathrm{p}<0.001, \eta^{2}=0.511$, was recorded. There was no group effect $(\mathrm{p}=0.064)$.

\section{Body image representation}

Based on statistical analysis, no statistically significant effect occurred: time* group $(\mathrm{p}=0.205)$, time $(\mathrm{p}=0.058)$, group $(\mathrm{p}=0.235)$.

\section{Coping strategies}

Higher scores were recorded on the positive coping strategies (positive refocusing and refocus on planning) in patients in the IVR group as compared to those in the kine- siotherapy group. However, there was an increase in the negative coping strategy (rumination) in the IVR patients compared to those in the kinesiotherapy condition.

Positive refocusing occurs when we think positive instead of thinking about our bad experiences. Studies show that positive refocusing strategy can be very beneficial to our wellbeing, while a low score on this strategy can be associated with a low level of emotional wellbeing. On this coping strategy, statistically significant results were obtained in terms of time* group interaction - $(1,10)=$ 23.456, $\mathrm{p}<0.001, \eta^{2}=0.566$, time $-\mathrm{F}(1,20)=17.618$, $\mathrm{p}=0.001, \eta^{2}=0,495$ and group $-\mathrm{F}(1,10)=4.849, \mathrm{p}=$ $0.041, \eta^{2}=0.212$.

Refocus on planning occurs whenever we think of the steps we need to take in order to deal with a negative event or whenever we think of a plan to change a straighten up things. A high score, coupled with action, is associated with enhanced wellbeing, while a low score is a sign of emotional distress. In this study, statistically significant results were recorded on the time*group interaction, F (1, $10)=23.529, \mathrm{p}<0.001, \eta^{2}=0.567$, as well as a time effect $\mathrm{F}(1,20)=28.471, \mathrm{p}<0.001, \eta^{2}=0.567$. There was no group effect $(\mathrm{p}=0.143)$.

Ruminating is the situation when we are obstinately thinking of and are concerned about feelings and thoughts related to a negative event. A high score on this strategy is associated with emotional problems or with psychopathological symptoms. In term of rumination, statistically significant results were recorded on the time* group interaction, $\mathrm{F}=6.661, \mathrm{p}=0.019, \eta^{2}=0.270$. There were no time effects $(p=0.60)$ and no group effects $(p=0.772)$ recorded.

\section{Pain correlations and psychological variables}

No significant correlations between phantom limb pain and the various psychological variables in post-intervention measurements were identified: depression symptoms $(\mathrm{r}=0.141)$, anxiety symptoms $(\mathrm{r}=0.067)$, pain catastrophizing $(r=-0.059)$. Likewise, no significant correlations were recorded between phantom limb pain and emotional and cognitive coping strategies: self-blame $(r=0.091)$, acceptance $(r=0.008)$, rumination $(r=-0.132)$, positive refocusing $(\mathrm{r}=0.046)(\mathrm{R}=-0.001)$, refocus of planning $(\mathrm{r}=$ $0.077)$, putting into perspective $(r=-0,194)$, catastrophizing $(r=-0,008)$, blaming others $(r=-0.256)$.

However, there were significant correlations recorded between anxiety symptoms and irrational pain catastrophizing thoughts $(r=0.545, \mathrm{p}<0.005$, two-tailed $)$ and coping strategies: refocus on planning $(r=-0.466, p$ $<0.005$, two-tailed $)$ and pain catastrophizing $(\mathrm{r}=0.784, \mathrm{p}$ $<0.001$, two-tailed).

\section{Discussions}

A variety of treatments have been historically used to alleviate phantom limb pain, with unsatisfied efficacy. Recently, immersive virtual reality has been employed as a more 
sophisticated mirror therapy. The purpose of this study was to investigate the extent to which an IVR-based intervention is more effective than an intervention based on kinesiotherapy in reducing phantom limb pain in patients with lower limb amputation. The results show that the IVR intervention is more effective, with pain alleviation in these patients recording significant levels. These results are consistent with those in some of the studies supporting the effectiveness of this method, though the major criticism is that such results come from case studies or single-subject experiments [28].

Moreover, the IVR-based intervention has also shown significant results in terms of psychological variables: increased quality of life, less irrational pain-related thoughts and positive coping strategies - positive refocusing and focus on planning. These data are very important, if we take into account the complex etiology of the pain in the phantom limb, and are underlining the positive psychological aspects that can be considered when designing multidisciplinary intervention programs.

Patients in the IVR group did not report significant decrease in their depression and anxiety symptoms, though a declining trend was seen in both groups. However, depression and anxiety symptoms in both groups recorded sub-clinical values. In addition, there were no significant concerns reported about the body image representation in the IVR patients compared to those in the kinesiotherapy group. These results are not consistent with those provided by the review conducted by Barbin et al. (2016), which suggests that an intervention based on a visual illusion of the missing limb, coupled with a virtual environment, may increase the amputee's concern about his or her body image.

Contrary to expectations, the IVR patients experienced a significant increase in the rumination coping strategy compared to those in the kinesiotherapy group. One possible explanation might be that this is a downside of the IVR therapy, which creates the illusion of an intact limb replacing the severed limb. IVR-based programs could take this aspect further into account and include psychological interventions that are specifically targeted to managing this particular kind of negative coping strategy. Again unlike expectations, depression symptoms appeared to be unrelated to the existence or intensity of pain, as it had been reported by some studies $[19,21]$. On the other hand, correlations were identified between symptoms of anxiety, negative pain-catastrophizing and negative coping strategies. In this regard, future IVR-based programs should consider interventions aimed at reducing anxiety in people with phantom limb pain.

One of the main limitations of this study is the small number of the study sample $(\mathrm{N}=20)$, which, though understandable for a pilot study on a new type of intervention, provides results that need to be treated with caution, being regarded as merely preliminary encouraging evidence. Future studies should validate these hypotheses on a larger and hence more representative sample. Moreover, future studies should assess the extent to which the results are replicable. Results should also be interpreted with caution, because the study did not include a passive control group of placebo or waiting list type.

\section{Conclusions}

The therapy based on immersive virtual reality (IVR) represents an efficient alternative in the management of phantom limb pain, achieving a significant reduction or even complete remission of pain. Through the positive impact IVR has upon the quality of life, coping, and irrational thinking, it increases the rehabilitation compliance and allows for an early recovery.

Across therapeutic disciplines, the evidence-base for treating phantom pain is fragile. Randomized controlled trials and systematic reviews are rare, and where they do exist, the conclusions are not encouraging. Recent progress includes the development of treatments, that directly target cortical mechanisms which have been linked to phantom pain. The initial data for IVR therapy is encouraging, but limited. The implementation of large, randomized, double-blinded, multicenter, placebo controlled trials, will be the focus of the future work.

\section{Conflict of interest}

None to declare.

\section{References}

1. Jensen TS, Rasmussen $P$ - Phantom pain and other phenomena after amputation. In: Wall PD, Melzack R, eds. Textbook of pain, 3rd ed. London: Churchill Livingstone, 1994:651- 683.

2. Ribbers G, Mulder T, Rijken R - The phantom phenomenon: a critical review. Int J Rehabil Res 1989;12:175-186.

3. Buchannan DC, Mandel AR - The prevalence of phantom limb experience in amputees. Rehabil Psychol 1986;31:183-188

4. Jensen TS, Krebs B, Nielsen J, Rasmussen P - Phantom limb, phantom pain and stump pain in amputees during the first six months following limb amputation. Pain 1983;17:243-256.

5. Parkes CM - Factors determining the persistence of phantom pain in the amputee. J PsychosomRes 1973;17:97-108.

6. Sherman RA, Sherman CJ, Parker L. Chronic phantom and stump pain among American veterans: results of a survey. Pain 1984;18:83-95.

7. Jensen TS, Krebs B, Nielsen J, Rasmussen P - Immediate and longterm phantom limb pain in amputees: incidence, clinical characteristics and relationship to pre-amputation limb pain. Pain. 1985 Mar;21(3):26778.

8. Manchikanti L, Singh V - Managing phantom pain. Pain Physician. 2004 Jul; $7(3): 365-75$.

9. Hansson PD, Anthony, Jensen, T - Pain 2008-an updated review refresher course syllabus : IASP Refresher Courses on Pain Management held in conjunction with the 12th World Congress on Pain, August 1722, 2008, Glasgow, Scotland. Castro-Lopes J, Raja S, Schmelz M, editors. Seattle: IASP Press; 2008.

10. Giummarra MJ, Moseley GL - Phantom limb pain and bodily awareness: current concepts and future directions. Current Opinion in Anesthesiology 2011,24:524-531

11. Halbert J, Crotty M, Cameron ID - Evidence for the optimal management of acute and chronic phantom pain: a systematic review. Clin J Pain 2002;18:84-92.

12. Schneider J, Hofmann A, Rost C, Shapiro F. EMDR in the treatment of chronic phantom limb pain. Pain Med 2008; 9:76-82.

13. Sharma RK, Singh B, Sharma S. Efficacy of Cognitive Behaviour Therapy and Quality Of Life in the Amputees. The International Journal of Indian Psychology. 2016; 3(3): 151-166. 
14. Murray C, Patchick E, Pettifer S, Caillette F, Phil D, Howard T Immersive Virtual Reality as a Rehabilitative Technology for Phantom Limb Experience: AProtocol.Cyberpsychology \& Behavior, 2006 9(2): 167-170.

15. Altschuler EL, Hu J - Mirror therapy in a patient with a fractured wrist and no active wrist extension. Scand J Plastic reconstr Surg Hand Surg 2008; 42: 110-111

16. Karmarkar A, Lieberman I - Mirror box therapy for complex regional pain syndrome. Anaesthesia 2006; 61:412-413.

17. Tichelaar Y, Geertzen JHB, Keizer D, van Wilgen CP. Mirror box therapy added to cognitive behavioral therapy in three chronic complex regional pain syndrome type I patients: a pilot study. Int J rehabil Res 2007; 30: 181-188.

18. Murray C, Pettifer S, Howard T, Patchick EL, Caillette F, Kulkarni J, Bamford C - The treatment of phantom limb pain using immersive virtual reality:Three case studies. Disability and Rehabilitation, September 2007; 29(18): 1465 - 1469.

19. Skoufa L, Givissis PK, Simos G. Pain Catastrophizing, Depression and Their Imact on Pain Intensity. International Journal of Novel Research in Healthcare and Nursing 2015; 2(3): 59-65.

20. Singh R, Ripley D, Pentland B, Todd I, Hunter J, Hutton L, Philip A. Depression and anxiety symptoms after lower limb amputation: the rise and fall. Clin Rehabil 2009 23: 281-286.
21. Desmond DM, MacLachlan M - Coping strategies as predictors of psychosocial adaptation in a sample of elderly veterans with acquired lower limb amputations. Social Science \& Medicine 2006; 62: 208-216

22. Jessie B, Seetha V, Casillas JM, Perennou D - The effects of mirror therapy on pain and motor control of phantom limb in amputees: A systematic review. Annals of physical and rehabilitation medicine 2016, http://dx.doiorg/101016/jrehab2016.04.001.

23. Melzack R. The McGill Pain Questionnaire: Major properties and scoring methods. Pain. 1975; 1: 277-299.

24. Sullivan MJL, Bishop SR, Pivkin J. The Pain Catastrophizing Scale: Development and validation. Psychological Assessment 1995; 7: 524532.

25. Breakey JW. Body Image: The Lower-Limb Amputee. J Prosthet Orthot 1997; 9: 58-66

26. Zigmond AS; Snaith RP. The hospital anxiety and depression scale. Acta Psychiatrica Scandinavica 1983; 67 (6): 361-370.

27. Garnefski N, Kraaij V, Spinhoven P. Negative life events, cognitive emotion regulation, and emotional problems. Personality and Individual Differences 2001; 30:1311-1327

28. Dunn J, Yeo E, Moghaddampour P, Chau B, Humbert S2. Virtual and augmented reality in the treatment of phantom limb pain: A literature review. NeuroRehabilitation. 2017; 40(4) : 595-601 